\title{
The World Space Observatory: ultraviolet mission: science program and status report
}

Sachkov, M., Gómez de Castro, A. I., Shustov, B.

M. Sachkov, A. I. Gómez de Castro, B. Shustov, "The World Space Observatory: ultraviolet mission: science program and status report," Proc. SPIE 11444, Space Telescopes and Instrumentation 2020: Ultraviolet to Gamma Ray, 1144473 (13 December 2020); doi: 10.1117/12.2562929 


\title{
The World Space Observatory - Ultraviolet mission: science program and status report
}

\author{
M. Sachkov ${ }^{* a}$, A.I. Gómez de Castro ${ }^{\mathrm{b}}$, B. Shustov ${ }^{\mathrm{a}}$ \\ ${ }^{a}$ Institute of Astronomy of Russian Academy of Sciences, Pyatnitskaya str., 48, Moscow, 119017, \\ Russia; \\ ${ }^{\mathrm{b}}$ AEGORA Research Group, Fac. CC. Matematicas, Universidad Complutense, Plaza de Ciencias 3, \\ 28040, Madrid, Spain
}

\begin{abstract}
A general description of the international space mission "Spectr-UF" (World Space Observatory-Ultraviolet) is given. The project is aimed to create a large space telescope to work in the UV wavelength domain (115 - $310 \mathrm{~nm})$. In the Federal Space Program of Russia for 2016-2025 the launch of the project is scheduled for 2025. We implement several important improvements: updated scientific program of the project; a new design of the Field Camera Unit instrument; corrected orbit; developed a system of collecting applications etc. This paper briefly describes the current status of the mission.
\end{abstract}

Keywords: ultraviolet, spectroscopy, imaging, exoplanet, UV space telescope, WSO-UV

\section{INTRODUCTION}

The Earth's atmosphere blocks observations of celestial objects in the ultraviolet (UV) part of the electromagnetic spectrum. Therefore, over the past half-century, many dozens of space UV experiments have been realized, mainly in the far and near UV wavelength range $91-310 \mathrm{~nm}$. Such observations provide important and unique, i.e. not obtained by other methods, scientific data leading to major astronomical discoveries such as direct detection of $\mathrm{H}_{2}$ molecules, discovery of the hot phase of the interstellar medium, determination of the relative content of deuterium to hydrogen, detection of the extended mode of star formation in galaxies, identification of the nature of structures (for example, rings of neutral gas, etc.) and processes occurring in the outer regions of the atmospheres of giant planets, detection of hydrogen crowns around exoplanets, etc (see a paper by Boyarchuk et al ${ }^{1}$.).

In 2019, the second astrophysical Observatory of the "Spektr" series of the Russian Federal Space Program was successfully launched (the first was the "Spektr-Radioastron" mission, 2011 -- 2019), which forms the basis of the astrophysical segment of the fundamental space research section of the Program. The "Spektr-RG" (X-ray Observatory) was launched into orbit in the vicinity of the L2 Lagrange libration point in the Sun-Earth system. The Observatory has been operating very successfully in space for more than a year. After that, the third project in the series - the "SpektrUF" project - became the next to be launched. All missions of the Spektr series use the same platform NAVIGATOR. We provide updated information on the WSO-UV project periodically in the SPIE meetings on specific systems/subsystems ${ }^{2,3,4}$. So, is it possible to track the development of the mission. Therefore, in this paper, we only briefly recall the main characteristics of the project and present its current status, focusing on recent changes. The main changes and clarifications made in the project include the following: updated scientific program of the project; selected another, optimal orbit; updated design of the cameras fields; considered extra scientific instrument - spectrograph; started the pilot collection of applications for the Project Core Program.

*msachkov@inasan.ru; phone+7-495-951-1279

Space Telescopes and Instrumentation 2020: Ultraviolet to Gamma Ray, edited by Jan-Willem A. den Herder Shouleh Nikzad, Kazuhiro Nakazawa, Proc. of SPIE Vol. 11444, 1144473 · (c) 2020 SPIE CCC code: $0277-786 X / 20 / \$ 21 \cdot$ doi: $10.1117 / 12.2562929$ 


\section{SCIENCE PROGRAM}

During the first two years of operation of the Spektr-UF Observatory, observation time will be divided between three areas: the Core program (approximately 50\% of the total time), National programs of participating countries (approximately $40 \%$ of the total observation time, in proportion to the financial contributions of countries to the project) and the Open program (open Program, implementation of applications of high scientific significance, including from scientists from countries not participating in the project). All proposals accepted under the Core program are large programs that require at least 100 hours of observation time.

First of all, the multi-purpose observatory WSO-UV will be used for the Core Program, the scientific justification of which, and the corresponding links are given in detail in the cited paper by Boyarchuk et al ${ }^{1}$. We will briefly recall the main elements of the Core program, giving a few explanatory examples and paying attention to new scientific accents. The Core observation program includes:

(1) Universe evolution studies. Several areas of research are included here, including the history of the reionization of the Universe, the chemical evolution of the Universe, and the search for hidden diffuse baryonic matter. We observe only $\sim 40--50 \backslash \%$ of the baryonic (ordinary) matter in the Universe. Where is the rest? This problem has been known for about 20 years. In recent years, a relatively new aspect has attracted particular attention, namely, that a similar problem arises when estimating the mass of heavy elements (metals): calculations of the expected mass of heavy elements that should be produced by stars in the Universe, starting from the first episodes of stellar nucleosynthesis and up to the current epoch, are noticeably higher than the observations of the contents of elements in galaxies and galaxy clusters. Here the hope for UV observations is justified, since they allow the most reliable determination of the content and spatial distribution of metals in the intergalactic environment and in galaxies.

(2) Studies of accretion processes. Physics of accretion on compact objects (black holes, neutron stars) and in close binary stars. In connection with the discovery of gravitational waves, there has been a sharp increase in interest in studying massive close binary stars - the progenitors of stellar-mass double black holes. Such stars emit mostly in the UV.

(3) Star formation studies. UV observations (images) of galaxies allowed us to highlight amazing facts about star formation in galaxies. It turns out that an amazing number of young stars are formed on the periphery of galaxies that do not show themselves in ordinary (non-UV images). This discovery was made with the help of the NASA GALEX mission, but there remains a large field of research on the fine structure and modes of star formation in galaxies.

(4) Studies of (exo)planetary atmospheres. There has been a growing interest in the possibilities of studying the hydrogen shells of exoplanets ${ }^{5}$ and the chemical composition of the atmospheres of these planets, as well as exocomets using UV instruments ${ }^{6}$. Given the large aperture and the instrument sensitivity, WSO-UV will be one of the major workhorse for exo- planet observations. The current exoplanet research can be divided into five major aspects: (i) planet detection, (ii) planet formation and migration, (iii) planets' physical properties, (iv) planets' chemical properties (including biomarkers), and (v) planets as a tool to study the properties of the host stars. The WSO-UV mission will be a crucial tool for the study of the last four aspects.

Because of the fact that preparatory observations are required for several proposals, WSO-UV Science Team decided to make a first Core Program Call for applications in 2018, several years before the launch date. The PI of such application should have a permanent position in astronomy/astrophysics in Russia or in Spain, countries that currently fund the WSO-UV project. The members of the team that make an application may include participants from any country.

On February 20, 2019, the Program Committee that was created with the involvement of international experts from Russia, Spain, Italy and France selected 17 projects from the received applications. Supported applications will make up approximately $20 \%$ of the time allocated to the Basic program. Research teams of supported projects received two years to conduct the necessary preparatory research and must submit a report on their implementation to the scientific management Committee of the Spektr-UF project. In 2021, the Committee will analyze these reports and draw up a final list of applications of the type discussed here for the Basic program. 
In 2018 the Institute of Astronomy of the Russian Academy of Science (INASAN) and the Universidad Complutense de Madrid (UCM) created and inaugurated the Joint Centre of UltraViolet Astronomy (JCUVA). The JCUVA hosts the facilities required for conducting the WSO-UV science operations run by Russia and Spain. Its activities also comprise the joint management of the WSO-UV science program.

\section{WSO-UV SCIENCE INSTRUMENTATION}

WSO-UV is a space astrophysical observatory for observations of objects in the Universe in the far and near UV range of the spectrum $(115-310 \mathrm{~nm})$. The Observatory is based on a complex of scientific instruments, which includes:

(1) The T-170M telescope (aperture $170 \mathrm{~cm}$, focal ratio 10 , field of view diameter 30 ', diffraction image quality in the center of the field). The telescope passed its vibration, static, transportation tests as well as thermal-vacuum tests. The T-170M telescope has inherited the successful experience gained during the Soviet ASTRON project $^{7}$.

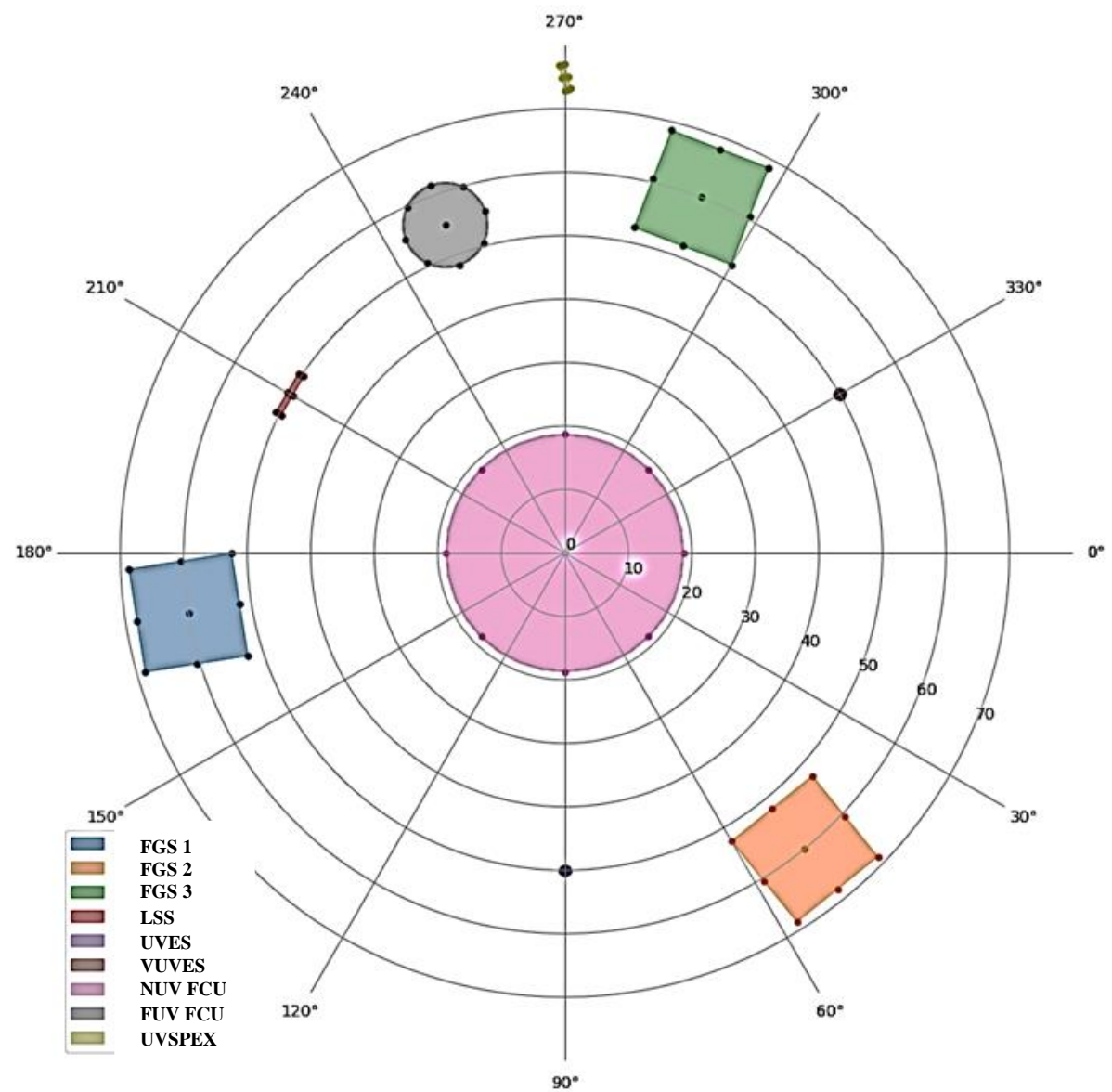

Figure 1. Projection of the fields of view of all instruments on the focal plane of the telescope. FGS - Fine Guidance Sensors; LSS - Long Slit Spectrograph; UVES - Ultraviolet Echelle Spectrograph; VUVES - Vacuum Echelle Spectrograph; NUV FCU - Focal Camera Unit Near UV Channel; FUV FCU - Focal Camera Unit Far UV Channel; UVSPEX - UV Spectrograph for Exoplanet Research 
(2) The WSO--UV spectrographs (WUVS) ${ }^{8}$. The instrument consists of a set of three instruments:

- The far UV high resolution spectrograph (VUVES) that will permit to carry out echellé spectroscopy with resolution about 50000 in the $115-176 \mathrm{~nm}$ range.

- The near UV high resolution spectrograph (UVES) to carry out echellé spectroscopy with resolution about 50 000 in the $174-310 \mathrm{~nm}$ range.

- The Long Slit Spectrograph (LSS) that will provide low resolution ( $R=1000)$, long slit spectroscopy in the $115-305 \mathrm{~nm}$ range. The spatial resolution will be 0.5 arcsec.

(3) The Field Camera Unit (FCU) ${ }^{9}$. FCU has two channels: The Near Ultraviolet channel covering wavelengths in the 170-310 nm interval and The Far Ultraviolet channel: working in the range 115-175 nm (see Fig. 3).

(4) The Ultraviolet Spectrograph for Exoplanets (UVSPEX). UVSPEX was proposed by Japan (Rykkyo University, see the paper by $\operatorname{Tavrov}^{10}$ et al.). The dominant engineering requirements for the UVSPEX as follows: the spectral resolution is better $0.5 \mathrm{~nm}$ to separate the O I line from other spectral lines. The spectral range is to exceed the wavelengths from $115 \mathrm{~nm}$ to $135 \mathrm{~nm}$ to detect at least $\mathrm{H}$ Lyman alpha $121.6 \mathrm{~nm}$ to O I 130 $\mathrm{nm}$. The throughput is better than $4 \%$. To achieve these requirements, a simple spectrograph design is proposed, containing the slit, the concave (toroid) grating as a dispersal element and the imaging photodetector. This optical concept is conventional and used in other space missions for UV spectroscopy.

The projection of the fields of view of all instruments on the focal plane of the telescope is shown on the Figure 1.

All spectrographs will be equipped with a CCD cooled to minus $100^{\circ} \mathrm{C}$. On the Fig 2 we present the image of the flight model of the detector.

The Conceptual design of the Field Camera Unit instrument is presented on the Fig 3.

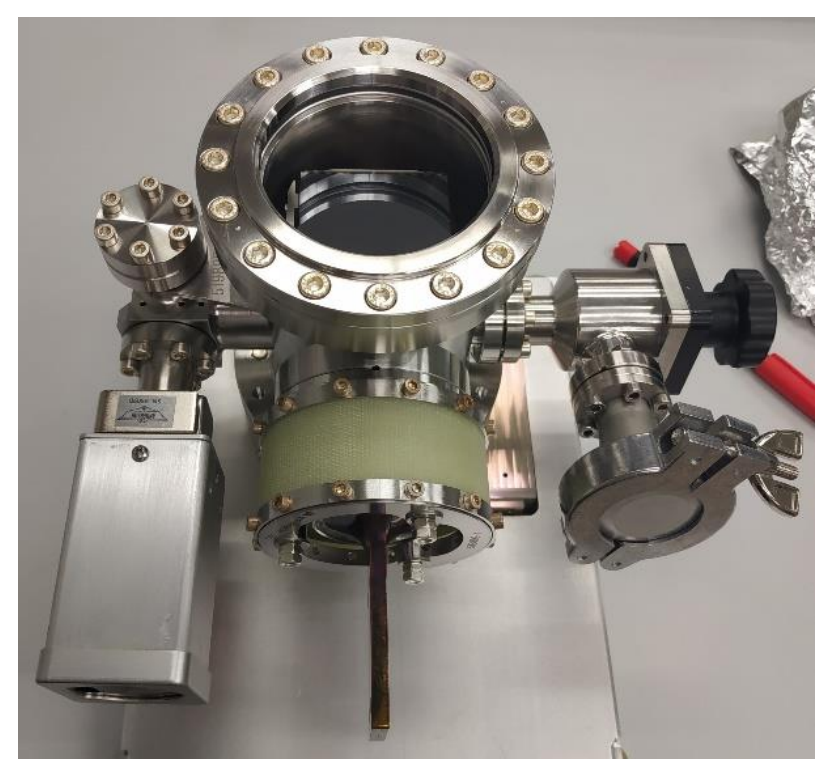

Figure 2. Detector of WSO-UV Spectrograph. 


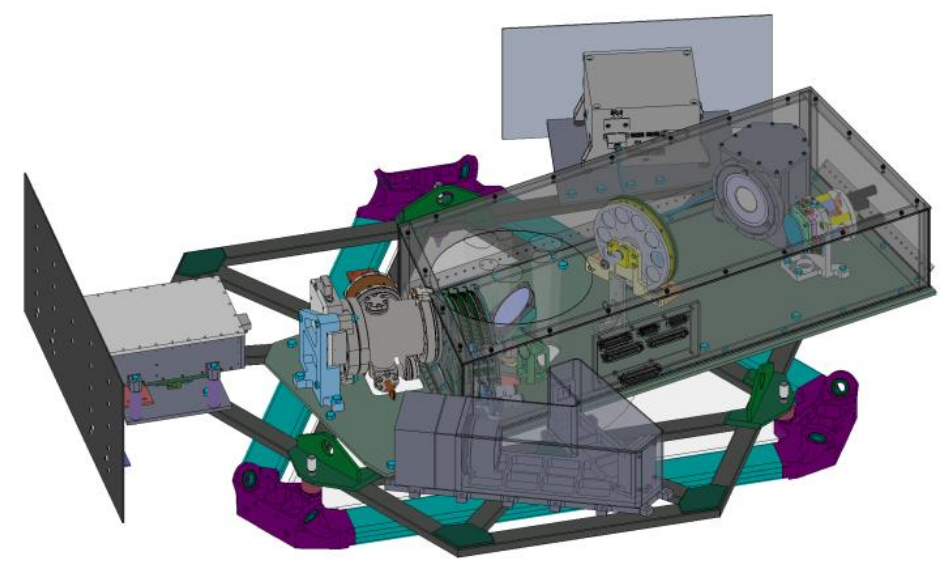

Figure 3. Conceptual design of the Field Camera Unit instrument.

\section{GROUND SEGMENT AND ORBIT}

WSO-UV will be launched to a geosynchronous orbit. There are several orbit selection criteria: the launcher capabilities, time in the Earth Radiation Belts, continuous visibility zones, time in the Earth shadow, available technical equipment of the Space and Ground Segments for radio communication. The orbit was selected to maximize the scientific return. WSO-UV high Earth orbit is also fundamental for monitoring programs and rapid follow-up observations of targets of opportunity. WSO-UV spacecraft will operate in the geosynchronous orbit with smaller inclination, 35 degrees instead of previously planned 51 degrees, to have access to the spacecraft from the Russian territory as long as possible. The new launcher ANGARA was proposed for WSO-UV. Ground Segment is comprised by all the infrastructure and facilities involved in the preparation and execution of the WSO-UV mission operations, which typically encompass real-time monitoring and control of the spacecraft, telescope and instruments as well as receiving, processing and storage of the scientific data. There will be two complete Science Operation Centers: the Russian one will be located in Moscow (Institute of Astronomy of the RAS), and the Spanish one that will be sited at Madrid (UCM).

\section{SUMMARY}

The Spektr-UF (WSO-UV) project is an efficient multipurpose orbital observatory for high and low resolution spectroscopy, high sensitivity imaging and slitless spectroscopy in the ultraviolet. It will open new opportunities in (exo)planetary science, extragalactic astronomy, stellar astrophysics, and cosmology. For exoplanetary studies a spectral resolution of $0.3 \mathrm{~nm}$ is required. It will be achievable by the WSO UV spectrographs, however, it is difficult to measure the weak stellar emission from low-temperature stars without a photon-counting detector. To realize exoplanet transit observations in oxygen spectral lines with the required accuracy for Earth-sized exoplanets, it was proposed a new instrument, the Ultraviolet Spectrograph for Exoplanets (UVSPEX). In the Federal Space Program of Russia 2016-2025 the launch of the project is scheduled for 2025.

Up to date information on the WSO-UV mission can be found on the web site of Joint Centre of Ultraviolet Astronomy: http://jcuva.space. 


\section{REFERENCES}

[1] Boyarchuk, A.A., et al., "Scientific problems addressed by the Spektr-UV space project (world space Observatory-Ultraviolet)," Astronomy Reports 60, 1 - 42 (2016)

[2] Sachkov, M., Sachkov, M., Shustov, B., \& Gómez de Castro, A.I., "Instrumentation of the WSO-UV project. DOI 10.1117/12.2055513," Proc. SPIE 9144, 914402 (2014)

[3] Sachkov, M., Shustov, B., Gómez de Castro, A. I., "World Space Observatory Ultraviolet mission: status 2016. DOI 10.1117/12.2233085," Proc. SPIE 9905, id. 990504, 6 pp. (2016)

[4] Sachkov, M., Shustov, B., Gómez de Castro, A. I., "World Space Observatory ultraviolet mission: instrumentation and the core program. DOI 10.1117/12.2312923," Proc. SPIE 10699, id. 106993G, 5 pp. (2018)

[5] Fossati, L., et al., "Major prospects of exoplanet astronomy with the World Space Observatory-UltraViolet mission," Astrophysics and Space Science 354, 9-19 (2014).

[6] Sachkov, M.E., "Spectral studies of comets in the ultraviolet range and prospects of the WSO-UV project in these studies," Solar System Research 50, 294 - 299 (2016)

[7] Boyarchuk, A., Savanov, I., Kanev, E., et al., "The Soviet ASTRON mission: legacy," Astrophys. Space Sci. 354, 247-250 (2014)

[8] Sachkov, M., et al., "Optical design of WUVS instrument: WSO-UV spectrographs. DOI," Proc. SPIE 9905, 990537-6 (2016)

[9] Sachkov, M., et al., "The new field camera unit imaging instrument onboard WSO-UV. DOI 10.1117/12.2312933," Proc. SPIE 10699, id. 1069935, 4 pp. (2018)

[10] Tavrov, A. et al., "Stellar imaging coronagraph and exoplanet coronal spectrometer: two additional instruments for exoplanet exploration onboard the WSO-UV 1.7-m orbital telescope," J. Astron. Telesc. Instrum., Syst. 4(4), 044001 (2018). 